\title{
Fixed-Rank Representation for Image Classification Using ANN and KNN
}

\author{
M. A. Zahed Javeed ${ }^{1}$, Prof: Shubhangi Sapkal ${ }^{2}$, Dr. R. R. Deshmukh ${ }^{3}$ \\ ${ }^{1}$ (Computer Science \& Engineering, Government College of Engineering, Aurangabad, India) \\ ${ }^{2}$ (Master of Computer Application, Government College of Engineering Aurangabad, India) \\ ${ }^{3}$ (Computer Science \& Engineering,Dr. Babasaheb Ambedkar Marathwada University Aurangabad, India)
}

\begin{abstract}
This study is focused on development an application of an efficient algorithm for subspace clustering and feature extraction that used for classification. Some previous techniques for subspace clustering make use of rank minimization and sparse based such as SSC and LRR are computationally expensive and may degrade clustering performance. So the need is to solve the problems of existing techniques, an algorithm is introduced known as Fixed-Rank Representation i.e. FRR based on matrix factorization is used for subspace clustering and feature extraction and that features are used for classification. For classification we use k-nearest neighbour and neural network classifiers and compare their accuracy or performance to classify the feature extracted from FRR.
\end{abstract}

Keywords: ANN, FRR, K-NN, LRR, SSC.

\section{Introduction}

In machine learning clustering and classification are two of the most important techniques for visual data analysis. In the last decade, there has been an increasing interest in sparsity to visual learning, such as object classification [1], image/video processing [2] and motion segmentation [3]. Early studies usually presented on unsupervised visual learning. Recently subspace clustering uses spars-based techniques such as spars subspace clustering (SSC) [4-5] and Low-Rank Representation [6-7]. Sparse Subspace Clustering i.e. SSC uses the 1D sparsest representation vectors produced by $l_{1}$-norm [8] minimization to define the affinity matrix of an undirected graph and subspace clustering is performed by spectral clustering techniques, such as normalized cut (NCut) [9]. However, as SSC computes the sparsest representation of each point individually, there is no constraint on the affinity matrix. This will reduce the clustering performance when data is grossly corrupted. Moreover SSC may over segment subspaces when dimension are more than 3-dimension.Low-Rank Representation (LRR) is another technique for spars based subspace clustering model. Low-Rank Representation is as same as Shape Interaction Matrix (SIM) [10] in absence of noise. LRR can perform true clustering when data sampling is sufficient and the subspaces are independent. However, LRR also have some limitations, first is the nuclear norm minimization in LRR typically requires calculating the singular value decomposition (SVD) at each iteration, which becomes computationally impractical as the problem is growing. Also if the observation is insufficient LRR may reduce clustering performance. Previously the FRR is applied to unsupervised visual learning. FRR parameterizes the representation matrix as the product of two low-rank matrices. FRR having some benefits over LRR, when there is no noise and the data samples are sufficient FRR is the optimal solution to LRR, we can say that FRR solve the multiple subspace structure. Moreover when the data samples are insufficient the memberships of samples to each subspace determined by FRR. Also the singular value decomposition (SVD) is the most expensive computational part of LRR, FRR avoids the SVD computation and it can be applied to large scale problem. In this study, we present the idea of matrix factorization with K-nearest neighbor [11] and neural network [12-13-18] into representation learning and improve functionality of Fixed-Rank Representation (FRR) to optimally solve the problems in supervised visual learning.

Following section II gives the idea of subspace clustering and classification is done, and section III contain experiment results and compare the performance of FRR using neural network and k-nearest neighbour for image classification in supervised learning is described.

\section{Subspace Clustering And Classification}

In this section we briefly describe process of subspace clustering and classification.

\subsection{Subspace Clustering}

Given a data set $X=X_{1}, X_{2}, \ldots, X_{K} \in \mathbb{R}^{D \times n}$ drawn from a union of $\mathrm{k}$ subspaces $\left\{C_{i}\right\}_{i=1}^{k}$ where $\mathrm{X}_{\mathrm{i}}$ is a collection of $n_{i}$ data points sampled from the subspace $C_{i}$ with an unknown dimension $d_{C_{i}}$. the goal of subspace 
clustering is to cluster data points into their respective subspaces. For subspace clustering we use the algorithm 1 given in [14-15] whose output is a graph constructed from the affine subspaces by using affinity $\left(\left|Z^{*}\right|+\left|\left(Z^{*}\right)^{\mathrm{T}}\right|\right)$ after that we apply the normalized cut (Ncut) algorithm. Where $Z$, is the spar set representation coefficient. The LRR i.e. Low-Rank Representation solves the minimization problem.

$$
\min _{Z}\|Z\| \text {., s.t. } X=X Z
$$

The idea behind FRR is to minimize the Frobenius norm of the representation $\mathrm{Z}$ instead of nuclear norm as in LRR FRR simultaneously computes a fixed lower rank representation $\breve{Z}$ we write $\operatorname{rank}(\breve{Z})=\mathrm{m}$. Obviously $\check{Z}$ can be expressed as $\check{Z}=\mathrm{LR}$, where $L \in \mathbb{R}^{n \times m}$ and ${ }^{R \in \mathbb{R}^{m \times n}}$. Replacing $\check{Z}$ by $L R$, we arrive at our basic FRR model.

$$
\begin{gathered}
\min _{Z, L, R}\|Z-L R\|_{F}^{2}, \\
\text { s.t. } \mathrm{X}=\mathrm{XZ}
\end{gathered}
$$

The normalization of $Z^{*}$ could be considered as a strong assumption, hence it cannot always be guaranteed in real situations. Therefore we can write equation (2) as

$$
\begin{gathered}
\min _{Z, L R}\|Z-L R\|_{F}^{2}, \\
\text { s.t., } X=X Z 1_{N}^{\tau}, Z=1_{N}^{\tau}
\end{gathered}
$$

In real application, the data is corrupted by both small noise ad gross outliers. To deal with corruptions as a new term $\mathrm{E}$, we consider the following regularized optimization problem.

$$
\begin{aligned}
& \min _{Z, L, E}\|Z-L R\|_{F}^{2}+\mu\|E\|_{S}, \\
& \text { s.t. } X=X Z+E, \quad 1_{n}^{\tau} Z=1_{n}^{\tau}
\end{aligned}
$$

Where the parameter $\mu>0$ is used to balance the effects of the two terms and $\|$. $\|_{\mathrm{s}}$ is a sparse norm corresponding to our assumption on $\mathrm{E}$. To get $\left(\mathrm{Z}^{*}, \mathrm{~L}^{*}, \mathrm{R}^{*}\right)$ we use the altering direction method i.e. ADM [16-17].

\subsection{Normalized Cuts.}

Normalized cut (N-Cut) by simply removing edges from two different parts of the graph and dissimilarity is calculated between two parts as total weight of edges that have been removed. In graph theory is known as cut. Fig. 1 indicates that minimizing the cut in the graph gives bad partitioning.

$$
\operatorname{cut}(A, B)=\sum_{u \in A v \in \bar{B}} w(u, v)
$$

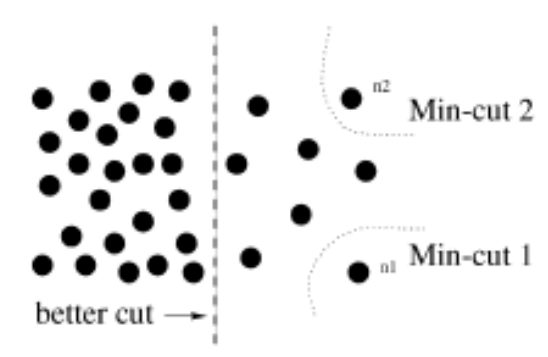

Fig.1. Minimum cut gives a bad portioning.

$\mathrm{Wu}$ and Leahy et. al. present a clustering method based on the minimum cut criteria. They partition the graph into k-sub graph such that maximum cut of sub graph is minimized. They compute the cut cost as the fraction of total edge connections to all nodes in the graph.

$$
\text { Ncut }=2-\operatorname{Nassoc}(A, B)
$$

The above equation gives the normalized cut in the graph. Hence, the tow partition criteria, minimization the disassociation in the groups and maximizing the association within the groups. After applying the ncut algorithm to the image we extract the features from the images using the FRR algorithm. FRR, will firstly resize the image in $32 \times 32$ and convert pixel values to $(0,1)$.

\subsection{Image classification}

There are many different methods for image classification but we study on supervised learning, so we use the most commonly algorithms, Feed-Forward Neural Network and K-Nearest Neighbor.

\subsubsection{Feed-Forward Neural Network:}

The pattern classification in neural network is best for linear associative network. But if the units in the output layer are nonlinear, then the network is limited by the linear separability constraint on the function relating the input-output pattern pairs. We need a way of updating these weights when each input-output pattern 
presented to network. To update the weight in supervisory mode, it is necessary to know desired output for each unit in the hidden and output layers. We used back propagation algorithm to solve this problem. Backpropagation algorithm is based on principle of gradient descendent along error surface. This approach is used to find the error (square difference between the desired output and the actual output obtained at the output layer of the network). The output is calculated using the current setting of the weights in all the layers. The optimum weights may be obtained if the weights are adjusted in such a way that the gradient descent is made along the total error surface. The objective is to determine the weight update for each presentation of an input-output pattern pair.

$$
\Delta w_{i j}(m)=-\eta \frac{\partial E(m)}{\partial w_{i j}}
$$

Where $\eta>=0$ is the a learning rate parameter, and to update the weight is given by

\subsubsection{K-Nearest Neighbor:}

$$
w_{i j}(m+1)=w_{i j}(m)+\Delta w_{i j}(m)
$$

The KNN classifier is based on non-parametric density estimation techniques. The KNN classifier is a very intuitive method, in the sense that it will classify based o their similarity with training data. For given given unlabeled example $\mathrm{x}_{\mathrm{u}} \varepsilon \mathcal{R}^{\mathrm{D}}$ find $\mathrm{k}$ closest label sample in the training data set and assign $\mathrm{x}_{\mathrm{u}}$ to class that appears most frequently in the k-subset.

Assume $x_{1}=x_{1}^{(1)}, x_{2}^{(2)}, \ldots, x_{1}^{d}$ is a d-dimensional feature vector of real umbers, for all $\mathrm{I} \mathrm{y}_{\mathrm{i}}$ is a class label in $\{1 \ldots C\}$, for all I our task is to determine $y^{\prime}$ for $x^{\prime} . K-N N$ finds the closest point to $x_{j}$ to $x^{\prime}$ with respect to Euclidean distance given by following equation, and classify the output according the $\mathrm{k}$ neighbor points.

$$
\left[a_{1}\left(x_{j}^{1}-x_{\text {new }}^{1}\right)^{2}+\ldots+a_{d}\left(x_{j}^{d}-x_{n e w}^{d}\right)^{2}\right]^{1 / 2}
$$

\section{Experiment And Result}

Previously the FRR applied on unsupervised visual data using normalized-cut (N-Cut) algorithm to obtain clusters. FRR can also be applicable to classify the supervised visual data i.e. image classification using k-nearest neighbor and neural network.

\subsection{Feature Extraction}

This experiment test the effectiveness of FRR for feature extraction for supervised data, and these feature extracted from FRR are used by ANN and KNN classifier for image classification. To simulate, we create a dataset by combining images with faces from face95 [19 ] and non face images from background [20]. We select 50 images of 50 different person or subjects from face95 database, having a total of 1900 images. From Caltech-background database, which contains 576 of gray color JPEG format images, we selected 50 images of each different background image. All images are resized to $32 \times 32$ and pixel values are normalized to $\{0,1\}$. Finally the goal is to robustly classify the images into two classes in presence of illumination and occlusion. Fig. 2 shows on left side the original image before applying the FRR algorithm and on right side the resized image into $32 \times 32$, and feature extracted image and also added error in the image

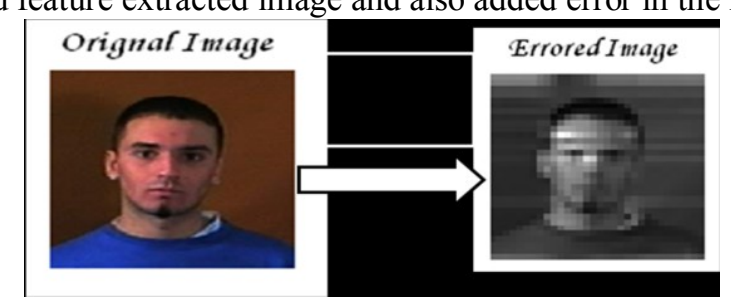

Fig.2. Original image and image, after applying FRR (error image).

\subsection{Image Classification}

Normally we have two classes 1 for face image and 2 for non-face image. We select the 40 images for testing from both classes and combine it into two different groups and train the neural network and k-nearest neighbor to classify these 40 images into two different classes. And finally we compare their accuracy by cross validating each of them and calculate their performance time to classify the 40 images. Figure 3 shows the performance graph of ANN and KNN. From this graph we clear that ANN require classification time is more than the KNN. The performance time calculated in millisecond. 


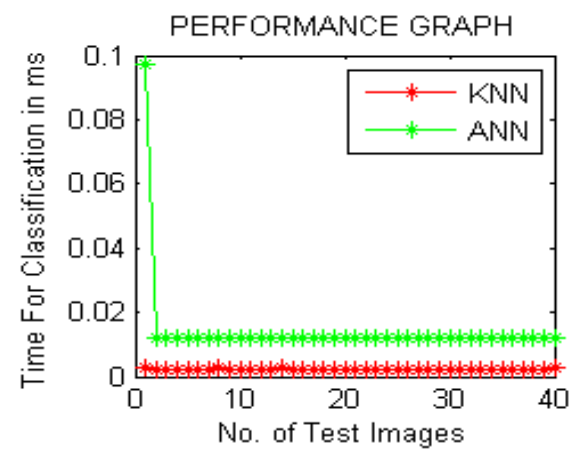

Fig.3. Performance time in millisecond of ANN and KNN.

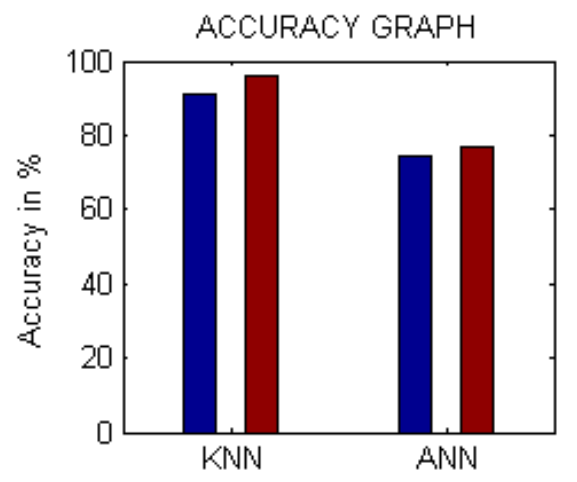

Fig.4. The graph shows classification accuracy of FRR by adding optimal error and error at first iteration. Accuracy in percentage, $\mathrm{KNN}$ gives $90.83 \%$ at optimal error term added in the images and $\mathbf{9 5 . 8 2 \%}$ at first iteration of FRR, and ANN has accuracy is about $74.16 \%$ at optimal error term find out by FRR and $76.67 \%$ at first iteration of FRR.

Table I

Table 1 shows the image names, class, and optimal error added in each images by FRR, and accuracy of FRR for image classification using ANN and KNN.

\begin{tabular}{|c|c|c|c|c|}
\hline Classifier & Image Names & Class & $\begin{array}{c}\text { Added Error In The Image } \\
\text { By Frr }\end{array}$ & Accuracy \\
\hline \multirow{3}{*}{ ANN } & 1.jpg & 2 & $9.96 \mathrm{E}-08$ & \multirow{3}{*}{$74.16 \%$} \\
\hline & 2.jpg & 2 & $9.97 \mathrm{E}-08$ & \\
\hline & 3.jpg & 1 & $9.91 \mathrm{E}-08$ & \\
\hline \multirow{2}{*}{ KNN } & 4.jpg & 1 & 9.99E-08 & \multirow{2}{*}{$90.83 \%$} \\
\hline & ardper.4.jpg & 1 & $9.96 \mathrm{E}-08$ & \\
\hline
\end{tabular}

\section{Conclusion}

The visual data like image, audio, video etc are become very massive and high dimensional, it is necessary to know how to extract compact knowledge from these data. The solution is to use idea of matrix factorization in to representation learning i.e. Fixed-Rank Representation and use them for classification. In this paper we learn FRR algorithm for supervised visual learning. FRR extract the feature from the give data set and these features are used by neural network and k-nearest neighbor to classify the images. The experiment on the two dataset given above is tested and compares the accuracy of FRR using KNN and ANN. KNN gives $90.83 \%$ at optimal error term added in the images and $95.82 \%$ at first iteration of FRR, and ANN has accuracy is about $74.16 \%$ at optimal error term find out by FRR and $76.67 \%$ at first iteration of FRR, so we can say that KNN is better than ANN tool for FRR for classification. Remain direction to Future work: Apply FRR for 3 or more dimensions, we can use FRR for object tracking and to provide a deeper analysis on LRR for subspace clustering.

\section{References}

[1] R. S. Cabral, F. De la Torre, J. P. costeria, ad A. Bermardio. Matrix completion for muti-label image classification. In NIPS, 2011.

[2] E.cades, X. Li, Y. Ma, and J. Wright. Robust principal component analysis. Joural of the ACM, 58(1): 1-37, 2011.

[3] S. Rao, R.Tron, R. Vidal, and Y. Ma. Motion segmentation in the presence of outlying, incomplete, and corrupted trajectories. IEEE Trans. on PAMI, 32(10):1832-1845, 2010.

[4] E. Elhamifar and R. Vidal. Sparse subspace clustering. In CVPR, 2009. 
[5] M. Soltanolkotabi and E. Cand 'es. A geometric analysis of subspace clustering with outliers. Technical Report (arXiv: 1112.4258), 2011.

[6] G. Liu, Z. Lin, and Y. Yu. Robust subspace segmentation by low-rank representation. In ICML, 2010.

[7] Y. Ni, J. Sun, X. Yan, and L. Cheong. Robust low-rank subspace segmentation with semi definite guarantees. In ICDM workshop, 2010 .

[8] Y. Albert Park. L1-norm Regularization. CSE 2914.2007.

[9] J. Shi and J. Malik. Normalized cuts and image segmentation. IEEE Trans. On PAMI, 22(8):888-905, 2000.

[10] J. Costeira and T. Kanade. A multibody factorization method for independently moving objcets. IJCV, 29(3):159-179, 1998.

[11] Xiaojin Zhu. K-nearest-neighbor an introduction to machine learning. Computer Sciences Department University of Wisconsin, Madison.

[12] Grish Kumar Jha. Artificial Neural Network. et. al. (2002).

[13] Dr. Roman V Belavkin. Feed Forward Neural Network. BIS3226.

[14] R. Liu, Z. Lin, F. De la Torre, and Z. Su. Fixed-rank representation for unsupervised visual learning. Technical Report (arXiv:1203.2210), 2012.

[15] R. Liu, Zhouchen Lin, Fernando De La Torre and Zhixun Su. Fixed-Rank Representation for unsupervised visual learning. IEEE 2012 8/12 ISBN 978-1-4673 1228/12.

[16] Z. Lin, M. Chen, L. Wu, and Y. Ma. The augmented Lagrange multiplier method for exact recovery of corrupted low-rank matrices. UIUC Technical Report UILU-ENG-09-2215, 2009.

[17] Y. Shen, Z. Wen, and Y. Zhang. Augmented Lagrangian alternating direction method for matrix separation based on low-rank factorization. preprint, 2011.

[18] Roya Asadi and Norwati Mustapha .New Supervised Multi Layer Feed Forward Neural Network Model to Accelerate Classification with High Accuracy. European Journal of Scientific Research ISSN 1450-216X Vol.33 No.1 (2009).

[19] Dr. Libor Spacek. Face Recognition Data (face 95). Computer vision Science Research Projects 2008.

[20] G.Griffin, A. Holub and P. Peoroa. Caltech-256 object category dataset. Caltech Technical Report, 2007. 